Scientific Research and Essays Vol. 6(16), pp. 3344-3350, 19 August, 2011

Available online at http://www.academicjournals.org/SRE

DOI: $10.5897 / \mathrm{SRE} 10.1088$

ISSN 1992-2248 @2011 Academic Journals

Full Length Research Paper

\title{
Finite-time stability analysis for linear time-varying singular impulsive systems
}

\author{
Linna Zhou ${ }^{1 *}$, Chunyu Yang ${ }^{2}$, Qingling Zhang ${ }^{3}$ and Yingwu Lai ${ }^{4}$ \\ ${ }^{1}$ School of Business Administration, Northeastern University, Shenyang, 110819, Peoples Republic of China. \\ ${ }^{2}$ State Key Laboratory of Synthetical Automation for Process Industries, Northeastern \\ University, Shenyang, 110819, Peoples Republic of China. \\ ${ }^{3}$ Institute of Systems Science, Northeastern University, Shenyang, 110819, Peoples Republic of China. \\ ${ }^{4}$ CNPC Liaoyang Petrochemical Company Machinary Factory, Liaoyang, 111003, Peoples Republic of China.
}

Accepted 28 July, 2011

\begin{abstract}
This paper considers the finite-time stability of linear time-varying singular impulsive systems. A lemma which states an important inequality was first established. Then some sufficient conditions for the systems to be finite-time stable were derived. The proposed results remove some restrictions of the existing methods and thus can be applied to more general systems. Finally, a numerical example was presented to illustrate the proposed approaches.
\end{abstract}

Key words: Singular impulsive systems, finite-time stability, comparison principle.

\section{INTRODUCTION}

Singular systems are also referred to as descriptor, semistate, implicit, constrained, differential-algebraic equation, or generalized state-space systems and arise naturally in many practical applications (Campbell, 1980). In the past several decades, many fundamental system theories developed for standard state-space systems have been successively generalized to its counterparts for singular systems (Ishihara and Terra, 2002; Cobb, 1984).

On the other hand, since impulsive behaviours which are characterized by abrupt changes of states at certain instants occur in many practical systems (Yang, 2001), impulsive systems have attracted particular interest (Zhang and Sun, 2005; Cheng et al., 2010; Wang and Liu, 2007). Recently, singular impulsive systems, that is, singular systems subject to impulsive effects, have been proposed and studied (Guan et al., 1995; 2001; 2005; Wang and Lia, 2001; Yao, 2006). The problems of stability and stabilization of singular impulsive systems

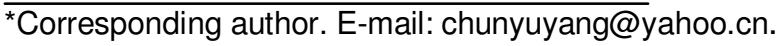
are studied in the framework of Lyapunov stability and the proposed methods are shown to be useful and efficient.

It is known that Lyapunov stability is concerned with the behavior of the system over an infinite time interval. In practice, a stable system might be useless because the stable domain or the domain of the desired attractor is not large enough and on the other hand, sometimes an unstable system may be acceptable since the system oscillates sufficiently near the desired state on a predefined finite time interval. This boundedness on a finite time interval is referred to as the notion of finite-time stability (or short-time stability) which is firstly introduced in La Salle and Lefschetz (1961). Different versions of finite-time stability for various systems have been proposed by Weiss and Infante (1967), Amato and Ariola (2001) and Liu and Sun (2008).

In Zhao et al. (2008), finite-time stability of linear timevarying singular impulsive systems is defined and some sufficient conditions are derived. The proposed methods make some restrictions on the systems under consideration including: (1) The derivative matrix $E(t)$ is 
nonsingular at each impulsive instant $\tau_{k}$; (2) The impulsive control matrix $B_{k}$ is symmetric and $I+B_{k}$ is nonsingular; (3) The involved disturbance signal is timeinvariant. In this paper, we aimed to study the finite-time stability of linear time-varying singular impulsive systems and try to remove the above mentioned restrictions. Thus, the development of new methods can be applied to more general systems.

\section{PRELIMINARIES}

The notations used in this paper are the same as those in Zhao et al. (2008). Let $R^{n}$ denote the $\mathrm{n}$-dimensional Euclidean space. $R^{+}=[0,+\infty)$ and $J=\left[t_{0}, t_{0}+T\right]$. The identity matrix of order $n$ is denoted by $I_{n}$ (or, simply, $I$ if no confusion arises). For any matrices $X, Y \in R^{n \times n}, \lambda_{\max }(Y, X)$ represents the maximum generalized eigenvalue of $(Y, X)$ (the generalized eigenvalues of $(Y, X)$ are defined by the solutions of $\operatorname{det}(s X-Y)=0$ and supposed to be real). When $X=I, \lambda_{\max }(Y, X)$ denotes the maximum eigenvalue of $X$ and is abbreviated as $\lambda_{\max }(Y)$.

We now recall the definitions and results of Zhao et al. (2008).

Definition 1: Time-varying matrix $E(t)$ is singular on time interval $J$, if there exists a $\tilde{t} \in J$ such that $\operatorname{det}(E(\tilde{t}))=0$.

Definition 2: Time-varying matrix $Q(t)$ is nonnegative definite on time interval $J$, if for all $\tilde{t} \in J, Q(\tilde{t})$ is nonnegative definite.

Consider the following singular impulsive system:

$$
\begin{cases}E(t) \dot{x}(t)=A(t) x(t)+\omega(t), & t \neq \tau_{k}, \\ \Delta x(t)=B_{k} x(t), & t=\tau_{k}, \\ x\left(t_{0}\right)=x_{0}, & k=1,2, \cdots,\end{cases}
$$

Where $t \in R^{+}, x(t) \in R^{n}$ is the state variable and $\omega(t) \in R^{n}$ is the disturbance signal. $A(t), E(t) \in R^{n \times n}$ are time-varying matrices and continuous with respect to $t$, and $B_{k} \in R^{n \times n}$. $E(t)$ is singular on $J . x_{0}$ is the initial value of the system state variable at $t_{0}$. A sequence $\left\{\tau_{k}, B_{k}\right\}$ has the effect of suddenly changing the state of System (1) at fixed points sequence $\left\{\tau_{k}\right\}$ with $\Delta x=x\left(\tau_{k}^{+}\right)-x\left(\tau_{k}^{-}\right)$,

Zhou et al. 3345
Where $x\left(\tau_{k}^{+}\right)=\lim _{h \rightarrow 0^{+}} x\left(\tau_{k}+h\right)$ and $x\left(\tau_{k}^{-}\right)=\lim _{h \rightarrow 0^{+}} x\left(\tau_{k}-h\right)$.

For simplicity, it is assumed that $x\left(\tau_{k}^{-}\right)=x\left(\tau_{k}\right)$. This means the state variable is left continuous at each $\tau_{k}$. Moreover, once the impulsive control $\left\{\tau_{k}, B_{k}\right\}$ for the linear singular system has been designed, the impulsive points $\tau_{k}$ are determined, not related with state variable $x$. For any state vector $x(t)$, quadratic form $\|x(t)\|_{Q}$ is defined as $\|x(t)\|_{Q}^{2}=x^{T}(t) Q(t) x(t)$, where $Q(t)=E^{T}(t) P(t) E(t), P(t)=P^{T}(t)>0 \quad$ is $\quad$ an arbitrarily specified matrix and continuous with respect to $t$.

It should be pointed out that this paper allows the disturbance signal $\omega(t)$ to be time-varying, while Zhao et al. (2008) requires it to be time-invariant.

Definition 3: Assume $\boldsymbol{a}(t) \equiv 0$. Then for given positive real numbers $c_{1}, c_{2}$ and nonnegative definite matrix $Q(t)$, system (1) is said to be finite-time stable with respect to $\left\{c_{1}, c_{2}, J, Q(t)\right\}$ if and only if:

$\left\|x\left(t_{0}\right)\right\|_{Q}^{2}<c_{1} \Rightarrow\left\|x\left(t_{0}\right)\right\|_{Q}^{2}<c_{2}, \forall t \in J$.

Definition 4: Given two positive real numbers $c_{1}, c_{2}$, a nonnegative definite matrix $Q(t)$ and a set $S \subset R^{n}$, system (1) is said to be finite-time stable with respect to $\left\{c_{1}, c_{2}, J, Q(t), S\right\}$ if and only if:

$\left\|x\left(t_{0}\right)\right\|_{Q}^{2}<c_{1} \Rightarrow\|x(t)\|_{Q}^{2}<c_{2}, \forall t \in J, \omega(t) \in S$.

The basic assumptions of Zhao et al. (2008) are stated as follows:

(H1) Time-varying matrices $A(t)$ and $E(t)$ are continuous on $J$.

(H2) $\lim _{k \rightarrow \infty} \tau_{k}=\infty$ and there exists $m$, such that:

$0 \leq t_{0}<\tau_{1}<\tau_{2}<\cdots<\tau_{m} \leq t_{0}+T<\tau_{m+1}<\cdots$.

(H3) For any $\tilde{t} \in J$, the pair $(E(\tilde{t}), A(\tilde{t}))$ is regular, that is, there exists a complex number $c$ such that $\operatorname{det}(c E(\tilde{t})-A(\tilde{t})) \neq 0$.

(H4) Matrices $B_{k}, k=1,2, \cdots m$ are symmetrical and $\operatorname{det}\left(I+B_{k}\right) \neq 0$.

(H5) For each $\tau_{k}, k=1,2, \cdots m, \operatorname{det}\left(E\left(\tau_{k}\right)\right) \neq 0$.
3346
Sci. Res. Essays 
(H6) Signal $\omega(t)$ is time-invariant and the set $S$ is defined as $S=\left\{\omega \mid \omega \in R^{n}, d \leq \omega^{T} \omega \leq h\right\}$, where $0<d \leq h$.

Theorem 1: (Zhao et al., 2008) Suppose $\boldsymbol{\alpha}(t) \equiv 0,(H 1)$-(H5) hold, $Q(t)=E^{T}(t) P(t) E(t) \geq 0$ and $P(t)=P^{T}(t)>0$. Then System (1) is finite-time stable with respect to $\left\{c_{1}, c_{2}, J, Q(t)\right\}$ if (H7) $c_{1} e^{\int_{t_{0}}^{\tau_{1}} \Lambda(M(t)) d t} \leq c_{2}$ and for each $0<k \leq m$, we have $c_{1} e^{\int_{t_{0}}^{\tau_{k}+1} \Lambda(M(t)) d t} \prod_{i=1}^{k} r_{i} \leq c_{2}, \forall t \in J$

Where:

$r_{k}=\lambda_{\text {max }}\left(\left(E^{T}\left(\tau_{k}\right) P\left(\tau_{k}\right) E\left(\tau_{k}\right)\right)^{-1}\left(I+B_{k}\right)^{T} E^{T}\left(\tau_{k}\right) P\left(\tau_{k}\right) E\left(\tau_{k}\right)\left(I+B_{k}\right)\right)$, $\Lambda(M(t))=\max \left\{x^{T}(t) M(t) x(t): x^{T}(t) Q(t) x(t)=1\right\}$,

and the matrix $M(t)$ is defined as:

$M(t)=\dot{E}^{T}(t) P(t) E(t)+A^{T}(t) P(t) E(t)+E^{T}(t) P(t) \dot{E}(t)+E^{T}(t) P(t) A(t)+E^{T}(t) \dot{P}(t) E(t)$.

Theorem 2: (Zhao et al., 2008) Suppose (H1)-(H6) hold, $Q(t)=E^{T}(t) P(t) E(t) \geq 0$ and $P(t)=P^{T}(t)>0$.

Then System (1) is finite-time stable with respect to $\left\{c_{1}, c_{2}, J, Q(t), S\right\}$ if there exist a constant $\alpha>0$ and a matrix $G=G^{T}>0$ such that:

$(\mathrm{H} 8)\left[\begin{array}{cc}M(t)-\alpha E^{T}(t) P(t) E(t) & E^{T}(t) P(t) \\ P(t) E(t) & -\alpha G\end{array}\right] \leq 0 ;$

(H9) $c \square e^{\alpha \tau_{1}}\left(c_{1}+\lambda_{\max }(G) h\right)-\lambda_{\min }(G) d \leq c_{2}$;

(H10) For any $2 \leq k \leq m$, we have $\alpha^{k-1} c+b \sum_{i=0}^{k-2} \alpha^{i} \leq c_{2}$, where $a=\max \left\{e^{\alpha\left(\tau_{k+1}-\tau_{k}\right)} r_{k}\right\}$,

$b=\max \left\{e^{\alpha\left(\tau_{k+1}-\tau_{k}\right)} \lambda_{\max }(G) h-\lambda_{\min }(G) d\right\}$,

$k=1,2, \cdots, m-1$, and $M(t)$ and $r_{k}$ are the same as those in Theorem 1.

\section{MAIN RESULTS}

It is known that one of the fundamental characteristics of singular systems is that the derivative matrix $E(t)$ is singular. However, assumption $(\mathrm{H} 5)$ requires $E(t)$ to be nonsingular at the impulse instant $\tau_{k}$. Assumption $(\mathrm{H} 4)$ is also a restrictive condition. The objective of this paper is to release the assumptions $(\mathrm{H} 4)$ and $(\mathrm{H} 5)$.

We first give the following lemma.

Lemma3 : Let $Q \in R^{n \times n}$ be a symmetric and semi positive definite matrix $P \in R^{n \times n}$ and be a symmetric matrix. Assume $\operatorname{rank}\left[\begin{array}{ll}P & Q\end{array}\right]=\operatorname{rank} Q$ and there exists a symmetric matrix $\bar{Q} \in R^{n \times n}$ such that $\operatorname{rank}[Q \bar{Q}]=n$ and $Q \times \bar{Q}=0$. Then the generalized eigenvalues of $(P+\bar{Q}, Q)$ are real and $P \leq \lambda_{\max }(P+\bar{Q}, Q) Q$.

Proof : Since $Q$ is a symmetric and semi-positive definite, there exists an orthogonal matrix $M$ such that $M^{T} Q M=\left[\begin{array}{ll}\Sigma & 0 \\ 0 & 0\end{array}\right]$, where $\Sigma>0$ is diagonal. Let $M^{T} P M=\left[\begin{array}{ll}P_{11} & P_{12} \\ P_{12}{ }^{T} & P_{22}\end{array}\right]$ and $M^{T} \bar{Q} M=\left[\begin{array}{cc}Q_{11} & Q_{12} \\ Q_{12}{ }^{T} & Q_{22}\end{array}\right]$.

Since $\operatorname{rank}[P Q]=\operatorname{rank} Q \quad, \quad$ we have $\operatorname{rank}\left[M^{T} P M \quad M^{T} Q M\right]=\operatorname{rank} M^{T} Q M$, which shows that

$\operatorname{rank}\left[\begin{array}{cccc}P_{11} & P_{12} & \Sigma & 0 \\ P_{12}{ }^{T} & P_{22} & 0 & 0\end{array}\right]=\operatorname{rank}\left[\begin{array}{cc}\Sigma & 0 \\ 0 & 0\end{array}\right]$. Then it follows from $\Sigma>0$ that $P_{12}=0, P_{22}=0$.

In addition, $\quad Q \times \bar{Q}=0 \quad$ implies $M^{T} Q M \times M^{T} \bar{Q} M=\left[\begin{array}{ll}\Sigma & 0 \\ 0 & 0\end{array}\right] \times\left[\begin{array}{cc}Q_{11} & Q_{12} \\ Q_{12}{ }^{T} & Q_{22}\end{array}\right]=\left[\begin{array}{cc}\Sigma Q_{11} & \Sigma Q_{12} \\ 0 & 0\end{array}\right]$.

Then it follows from $\Sigma>0$ that $Q_{11}=0, Q_{12}=0$

Since $\operatorname{rank}[Q \bar{Q}]=n \quad, \quad$ we have $n=\operatorname{rank}[Q \bar{Q}]=\operatorname{rank}\left[M^{T} Q M \quad M^{T} \bar{Q} M\right]$

$=\operatorname{rank}\left[\begin{array}{cccc}\Sigma & 0 & Q_{11} & Q_{12} \\ 0 & 0 & Q_{12}{ }^{T} & Q_{22}\end{array}\right]$

$=\operatorname{rank}\left[\begin{array}{cccc}\Sigma & 0 & 0 & 0 \\ 0 & 0 & 0 & Q_{22}\end{array}\right]$

Which yields that $Q_{22}$ is nonsingular.

Then we have: 


$$
\begin{aligned}
& \operatorname{det}(s Q-(P+\bar{Q}))=0 \Leftrightarrow \operatorname{det}\left(s M^{T} Q M-M^{T}(P+\bar{Q}) M\right)=0 \\
& \Leftrightarrow \operatorname{det}\left(s\left[\begin{array}{ll}
\Sigma & 0 \\
0 & 0
\end{array}\right]-\left[\begin{array}{cc}
P_{11} & 0 \\
0 & Q_{22}
\end{array}\right]\right)=0 \\
& \Leftrightarrow \operatorname{det}\left(\left[\begin{array}{cc}
s \Sigma-P_{11} & 0 \\
0 & -Q_{22}
\end{array}\right]\right)=0 \\
& \Leftrightarrow \operatorname{det}\left(s \Sigma-P_{11}\right)=0 \\
& \Leftrightarrow \operatorname{det}\left(s I-\Sigma^{-\frac{1}{2}} P_{11} \Sigma^{-\frac{1}{2}}\right)=0 \text {. }
\end{aligned}
$$

Since $\Sigma^{-\frac{1}{2}} P_{11} \Sigma^{-\frac{1}{2}}$ is symmetric, the eigenvalues of $\Sigma^{-\frac{1}{2}} P_{11} \Sigma^{-\frac{1}{2}}$ are real. Then it follows from (3) that the generalized eigenvalues of $(P+\bar{Q}, Q)$ are real.

Furthermore, from Equation (3), we have $\Sigma^{-\frac{1}{2}} P_{11} \Sigma^{-\frac{1}{2}} \leq \lambda_{\max }\left(\Sigma^{-\frac{1}{2}} P_{11} \Sigma^{-\frac{1}{2}}\right) I=\lambda_{\text {max }}(P+\bar{Q}, Q) I$, which shows that $P_{11} \leq \lambda_{\max }(P+Q, Q) \Sigma$

.Then

$M^{T} P M \leq \lambda_{\max }(P+\bar{Q}, Q)\left[\begin{array}{ll}\Sigma & 0 \\ 0 & 0\end{array}\right]$, which is equivalent to $P \leq \lambda_{\max }(P+\bar{Q}, Q) Q$. This completes the proof.

Remark 1: From Equation (3), the generalized eigenvalues of $(P+\bar{Q}, Q)$ is independent of the choice of $Q$ satisfying $\operatorname{rank}[Q \bar{Q}]=n$ and $Q \times \bar{Q}=0$.

As shown in Zhao et al. (2008), under the assumptions $(\mathrm{H} 4)$ and $(\mathrm{H} 5)$ and choosing $r_{k}$ as $(\mathrm{H} 7)$, one has:

$$
\begin{aligned}
& x^{T}\left(\tau_{k}\right)\left(I+B_{k}\right)^{T} E^{T}\left(\tau_{k}\right) P\left(\tau_{k}\right) E\left(\tau_{k}\right)\left(I+B_{k}\right) x\left(\tau_{k}\right) \\
\leq & r_{k} x^{T}\left(\tau_{k}\right) E^{T}\left(\tau_{k}\right) P\left(\tau_{k}\right) E\left(\tau_{k}\right) x\left(\tau_{k}\right)
\end{aligned}
$$

Which plays an important role in the proofs for Theorems 1 and 2.

Let $X_{k}=E^{T}\left(\tau_{k}\right) P\left(\tau_{k}\right) E\left(\tau_{k}\right)$

And

$$
Y_{k}=\left(I+B_{k}\right)^{T} E^{T}\left(\tau_{k}\right) P\left(\tau_{k}\right) E\left(\tau_{k}\right)\left(I+B_{k}\right)
$$

We now introduce the following assumptions:

(H11) $\operatorname{rank}\left[Y_{k} X_{k}\right]=\operatorname{rankX}_{k}$ and there exists a Zhou et al. $\quad 3347$ symmetric matrix $\bar{X}_{k} \in R^{n \times n} \quad$ such that $\operatorname{rank}\left[X_{k} \bar{X}_{k}\right]=n$ and $X_{k} \times \bar{X}_{k}=0$.

We now define $r_{k}$ as:

$r_{k}=\lambda_{\max }\left(Y_{k}+\bar{X}_{k}, X_{k}\right)$

From Lemma 3, it can be seen that Inequality (4) holds for $r_{k}$ defined by Equation (7) if $(\mathrm{H} 11)$ holds. Then, similarly to the proofs for Theorems 1 and 2 given in Zhao et al. (2008), one can prove the following Theorems 4 and 5.

Theorem 4 : Suppose $\omega(t) \equiv 0,(\mathrm{H} 1)-(\mathrm{H} 3)$ and $(\mathrm{H} 11)$ hold, $P(t)=P(t)^{T}>0$ and $Q(t)=E(t)^{T} P(t) E(t) \geq 0$. Then System (1) is finite-time stable with respect to $\left\{c_{1}, c_{2}, J, Q(t)\right\}$ if $\left(\mathrm{H}^{\prime}{ }^{\prime}\right) \quad c_{1} e^{\int_{\tau_{0}}^{\tau_{1}} \Lambda(M(t)) d t} \leq c_{2}$ and for each $0<k \leq m$, we have:

$$
c_{1} e^{\int_{t_{0}}^{\tau_{k}+1} \Lambda(M(t)) d t} \prod_{i=1}^{k} r_{i} \leq c_{2}, \forall t \in J
$$

Where $r_{k}$ is defined by (7), matrix $M(t)$ and $\Lambda(M(t))$ are the same as those in Theorem 1.

Theorem 5 : Suppose $(\mathrm{H} 1)-(\mathrm{H} 3),(\mathrm{H} 6)$ and $(\mathrm{H} 11)$ hold, $P(t)=P(t)^{T}>0$ and $Q(t)=E(t)^{T} P(t) E(t) \geq 0$. Then System (1) is finite-time stable with respect to $\left\{c_{1}, c_{2}, J, Q(t), S\right\}$ if there exist a constant $\alpha>0$ and a matrix $G=G^{T}>0$ such that (H8), (H9) and (H10 ') For any $2 \leq k \leq m$, we have $a^{k-1} c+b \sum_{i=0}^{k-2} a^{i} \leq c_{2}$, where $r_{k}$ is defined by Equation (7), $a=\max \left\{e^{\alpha\left(\tau_{k+1}-\tau_{k}\right)} r_{k}\right\}$, $b=\max \left\{e^{\alpha\left(\tau_{k+1}-\tau_{k}\right)} \lambda_{\text {max }}(G) h-\lambda_{\text {min }}(G) d\right\}, k=1,2, \cdots m-1$, and $M(t)$ is the same as that in Theorem 4.

Remark 2 : The newly developed Theorems 4 and 5 are less restrictive than Theorems 1 and 2, respectively. Assume that (H5) holds. Then $X_{k}$ is nonsingular, which implies that $\operatorname{rank}\left[\begin{array}{ll}Y_{k} & X_{k}\end{array}\right]=\operatorname{rank} X_{k}$.

Choosing $\quad \bar{X}_{k}=0_{n \times n} \quad$,one gets $\operatorname{rank}\left[X_{k} \bar{X}_{k}\right]=n$ 
and $X_{k} \times \bar{X}_{k}=0$. Thus we have that assumption (H5) $3348 \quad$ Sci. Res. Essays

implies $(\mathrm{H} 11)$. Furthermore, since the eigenvalues of $X_{k}^{-1} Y_{k}$ are the same as the generalized eigenvalues of $\left(Y_{k}, X_{k}\right)$, the $r_{k}$ defined in Theorem 1 is the same to that defined by Equation (7). Hence, Theorems 4 and 5 reduce to Theorems 1 and 2, respectively, when assumption (H5) holds.

In practice, the disturbance signal is usually timevarying. However, the assumptions in Theorems 2 and 5 require the disturbance signal to be time-invariant. We now consider the finite-time stability of singular impulsive systems with time-varying disturbance. To do this, we make the following assumption:

$\left(\mathrm{H} 6{ }^{\prime}\right)$ Signal $\omega(t)$ is time-varying and the set $S$ is defined as $S=\left\{\omega \mid \omega \in R^{n}, 0 \leq \omega^{T} \omega \leq h\right\}$, where $h>0$.

Theorem 6: Suppose $(\mathrm{H} 1)-(\mathrm{H} 3),\left(\mathrm{H}^{\prime}\right)$ and $(\mathrm{H} 11)$ hold, $P(t)=P(t)^{T}>0$ and $Q(t)=E(t)^{T} P(t) E(t) \geq 0$. Then System (1) is finite-time stable with respect to $\left\{c_{1}, c_{2}, J, Q(t), S\right\}$ if there exist a constant $\alpha>0$ and a matrix $G=G^{T}>0$ such that $(\mathrm{H} 8)$ and $(\mathrm{H} 12)$ $c:=e^{\alpha \tau_{1}}\left(c_{1}+\lambda_{\text {max }}(G) h\right)-\lambda_{\text {min }}(G) d \leq c_{2} \quad(\mathrm{H} 13) \quad$ For any $2 \leq k \leq m$ we have $\alpha^{k-1} c+b \sum_{i=0}^{k-2} \alpha^{i} \leq c_{2}$, where $r_{k}$ is defined by Equation $b=\max \left\{e^{\alpha\left(\tau_{k+1}-\tau_{k}\right)} \lambda_{\text {max }}(G) h-\lambda_{\text {max }}(G) h\right\}$, $a=\max \left\{e^{\alpha\left(\tau_{k+1}-\tau_{k}\right)} r_{k}\right\}, k=1,2, \cdots, m-1$, and $M(t)$ is the same as that in Theorem 1.

Proof : Choose the Lyapunov function $V(t, x(t))=x^{T}(t) E^{T}(t) P(t) E(t) x(t)$.

Consider the situation on the interval $t \in\left(t_{0}, \tau_{1}\right)$, one has:

$\dot{V}(t, x(t))=x^{T}(t) M(t) x(t)+\omega^{T}(t) P(t) E(t) x(t)+x^{T}(t) E^{T}(t) P(t) \omega(t)$.

From (H8), it follows that:

$$
\begin{aligned}
& {\left[\begin{array}{l}
x(t) \\
\omega(t)
\end{array}\right]^{T}\left[\begin{array}{cc}
M(t)-\alpha E^{T}(t) P(t) E(t) & E^{T}(t) P(t) \\
P(t) E(t) & -\alpha G
\end{array}\right]\left[\begin{array}{c}
x(t) \\
\omega(t)
\end{array}\right]} \\
& =x^{T}(t) M(t) x(t)+\omega^{T}(t) P(t) E(t) x(t)+x^{T}(t) E^{T}(t) P(t) \omega(t) \\
& \quad-\alpha x^{T}(t) E^{T}(t) P(t) E(t) x(t)-\alpha \omega(t) G \omega(t) \\
& \leq 0
\end{aligned}
$$

By Equations (8) and (9), we have:

$$
\begin{aligned}
\dot{V}(t, x(t)) & \leq \alpha V(t, x(t))+\alpha \omega^{T}(t) G \omega(t) \\
& \leq \alpha V(t, x(t))+\alpha \lambda_{\max }(G) h .
\end{aligned}
$$

Then, using comparison principle (Pachpatte, 1998), we have:

$V(t, x(t)) \leq e^{\alpha\left(t t_{0}\right)}\left(V\left(t_{0}, x\left(t_{0}\right)\right)+\lambda_{\max }(G) h\right)-\lambda_{\max }(G) h, \forall t \in\left(t_{0}, \tau_{1}\right)$.

It follows from Equation (11) and (H12) that $\left\|x\left(t_{0}\right)\right\|_{Q}^{2}<c_{1} \Rightarrow\|x(t)\|_{Q}^{2}<c_{2}, \forall t \in\left(t_{0}, \tau_{1}\right)$.

Since $x\left(\tau_{1}^{-}\right)=x\left(\tau_{1}\right)$ and $\tau^{-} \in\left(t_{0}, \tau_{1}\right)$, Inequality implies:

$V\left(\tau_{1}, x\left(\tau_{1}\right)\right) \leq e^{\alpha\left(\tau_{1}-t_{0}\right)}\left(V\left(t_{0}, x\left(t_{0}\right)\right)+\lambda_{\max }(G) h\right)-\lambda_{\max }(G) h$

which shows $\left\|x\left(t_{0}\right)\right\|_{Q}^{2}<c_{1} \Rightarrow\left\|x\left(\tau_{1}\right)\right\|_{Q}^{2}<c_{2}$. Then $\left\|x\left(t_{0}\right)\right\|_{Q}^{2}<c_{1} \Rightarrow\|x(t)\|_{Q}^{2}<c_{2}, \forall t \in\left(t_{0}, \tau_{1}\right]$.

We now consider the case $t \in\left(\tau_{2}, \tau_{2}\right)$, on which Equation (10) also holds.

Using comparison principle again, we have:

$V(t, x(t)) \leq e^{\alpha\left(t-\tau_{1}\right)}\left(V\left(\tau_{1}^{+}, x\left(\tau_{1}^{+}\right)\right)+\lambda_{\text {tax }}(G) h\right)-\lambda_{\text {max }}(G) h, \forall t \in\left(\tau_{1}, \tau_{2}\right)$.

From $(\mathrm{H} 1),(\mathrm{H} 11),(\mathrm{H} 13)$ and Lemma 3, we have:

$$
\begin{aligned}
V\left(\tau_{1}^{+}, x\left(\tau_{1}^{+}\right)\right)= & x^{T}\left(\tau_{1}^{+}\right) E^{T}\left(\tau_{1}\right) P\left(\tau_{1}\right) E\left(\tau_{1}\right) x^{T}\left(\tau_{1}^{+}\right) \\
& =x^{T}\left(\tau_{1}\right)\left(I+B_{k}\right)^{T} E^{T}\left(\tau_{1}\right) P\left(\tau_{1}\right) E\left(\tau_{1}\right)\left(I+B_{k}\right) x\left(\tau_{1}\right) \\
& \leq r_{k} x^{T}\left(\tau_{1}\right) E^{T}\left(\tau_{1}\right) P\left(\tau_{1}\right) E\left(\tau_{1}\right) x\left(\tau_{1}\right) \\
& =r_{k} V\left(\tau_{1}, x\left(\tau_{1}\right)\right) .
\end{aligned}
$$

Thus it follows from Inequalities (13) and (14) that:

$$
V(t, x(t)) \leq e^{\alpha\left(t-\tau_{1}\right)}\left(r_{k} V\left(\tau_{1}, x\left(\tau_{1}\right)\right)+\lambda_{\text {max }}(G) h\right)-\lambda_{\text {max }}(G) h, \forall t \in\left(\tau_{1}, \tau_{2}\right]
$$


By the same progress, for $k=2,3, \cdots, m$, we have:

$$
\begin{aligned}
V(t, x(t)) & \leq e^{\alpha\left(\tau_{k+1}-\tau_{k}\right)}\left(r_{k} V\left(\tau_{k}, x\left(\tau_{k}\right)\right)+\lambda_{\max }(G) h\right)-\lambda_{\max }(G) h \\
& \leq a V\left(\tau_{k}, x\left(\tau_{k}\right)\right)+b \\
& \leq d^{k-1} V\left(\tau_{1}, x\left(\tau_{1}\right)\right)+b \sum_{i=0}^{k-2} a^{i}, \forall t \in\left[\tau_{k}, \tau_{k+1}\right] .
\end{aligned}
$$

$$
\begin{aligned}
& \text { Then by } \quad \text { (H13), we } \\
& \left\|x\left(t_{0}\right)\right\|_{Q}^{2}<c_{1} \Rightarrow\|x(t)\|_{Q}^{2}<c_{2}, \forall t \in\left(\tau_{k}, \tau_{k+1}\right] .
\end{aligned}
$$

This completes the proof.

Remark 3 : It is easy to show that Theorem 6 still holds if the time-varying disturbance $\omega(t)$ in system (1) is replaced by a bounded nonlinear function $f(x, t)$ satisfying $f^{T}(x, t) f(x, t) \leq h$. Thus Theorem 6 also describes a finite-time stability analysis method for a class of nonlinear singular impulsive systems.

\section{NUMERICAL EXAMPLE}

Consider System (1) with:

$E(t)=\left[\begin{array}{cc}1 & 0 \\ 0 & \sin (0 D-t)\end{array}\right], A(t)=\left[\begin{array}{cc}1 & 0 \\ 0 & \cos (O D-t)\end{array}\right],(t)=\left[\begin{array}{l}O O B \\ O C D\end{array}\right] \sin (t), B=\left[\begin{array}{cc}-2 & 0 \\ 1 & -1\end{array}\right] x(0)=\left[\begin{array}{l}1 \\ 2\end{array}\right]$.

Let $\quad J=\left[\begin{array}{ll}0, & 0.1\end{array}\right], h=0.0045, c_{1}=1, c_{2}=2.5 \quad$ and $\tau_{k}=0.05 k, k=1,2$.

Since $B_{k} \neq B_{k}{ }^{T}$ and $E\left(\tau_{1}\right)=\left[\begin{array}{ll}1 & 0 \\ 0 & 0\end{array}\right]$ is singular, assumptions $(\mathrm{H} 4)$ and $(\mathrm{H} 5)$ do not hold.

Furthermore, the disturbance is time-varying, which shows that $(\mathrm{H} 6)$ does not hold.

Thus Theorems 2 and 4 cannot be used in this example. We will use Theorem 6 to study the finite-time stability of the system.

It can be seen that: the matrices $A(t)$ and $E(t)$ are continuous on $J ; \lim _{k \rightarrow \infty} \tau_{k}=\infty$ and for $\mathrm{m}=2$, it holds that $\quad 0 \leq t_{0}<\tau_{1}<\tau_{2} \leq 0.1 \quad ; \quad$ For any $\tilde{t} \in J, \operatorname{det}(c E(\tilde{t})-A(\tilde{t})) \neq 0 ; \omega^{T}(t) \omega(t) \leq h$. Thus assumptions $(\mathrm{H} 1)-(\mathrm{H} 3)$ and $\left(\mathrm{H} 66^{\prime}\right)$ hold.

Let $P=I, G=2 I, \alpha=8$. Then:
$X_{1}=\left[\begin{array}{ll}1 & 0 \\ 0 & 0\end{array}\right], Y_{1}=\left[\begin{array}{ll}1 & 0 \\ 0 & 0\end{array}\right], X_{2}=\left[\begin{array}{cc}1 & 0 \\ 0 & \sin ^{2}(005)\end{array}\right], Y_{2}=\left[\begin{array}{cc}-1+\sin ^{2}(005) & 0 \\ 0 & 0\end{array}\right], M(t)=\left[\begin{array}{ll}4 & 0 \\ 0 & 0\end{array}\right]$.

Choosing $\quad \bar{X}_{1}=\left[\begin{array}{ll}0 & 0 \\ 0 & 1\end{array}\right], \bar{X}_{2}=\left[\begin{array}{ll}0 & 0 \\ 0 & 0\end{array}\right]$, we have that assumption ( $\mathrm{H} 11)$ holds.

It can be verified that;

Zhou et al.

3349

$\left[\begin{array}{cccc}-4 & 0 & 1 & 0 \\ 0 & -8 \sin ^{2}(0.05-t) & 0 & \sin (0.05-t) \\ 1 & 0 & -16 & 0 \\ 0 & \sin (0.05-t) & 0 & -16\end{array}\right] \leq 0_{\text {which }}$

shows that $(\mathrm{H} 8)$ holds.

By

simple calculation,

$r_{1}=1, r_{2}=0, \lambda_{\max }(G)=2, a=1.4918, b=0.0044, c=1.4963$. Then $H(12)$ and $H(13)$ hold. From Theorem 6 , this system is finite-time stable with respect to $\left\{c_{1}, c_{2}, J, Q(t), S\right\}$ with

$J=[0,0.1], c_{1}=1, c_{2}=2.5, S=\left\{\omega \mid \omega^{T}(t) \omega(t) \leq 0.0045\right\}$

and $Q(t)=\left[\begin{array}{cc}1 & 0 \\ 0 & \sin ^{2}(0.05-t)\end{array}\right]$.

\section{Conclusion}

This paper has investigated the finite-time stability of linear time-varying singular impulsive systems. New sufficient conditions for the system to be finite-time stable have been derived by a newly developed inequality and the well-known comparison principle. Compared with the existing results, the proposed methods remove some basic assumptions and therefore can be applied to more general systems. The presented numerical example has illustrated the obtained results.

\section{ACKNOWLEDGMENTS}

This work was supported by the Natural Science Foundation of China (60904009), the Fundamental Research Funds for the Central Universities of China (N100406010,N090408001), the Funds for Creative Research Groups of China (60821063) and the National Basic Research Program of China (2009CB3206 01). 


\section{REFERENCES}

Amato F, Ariola M, Dorato P (2001). Finite-time control of linear systems subject to parametric uncertainties and disturbances. Automatica, 37(9): 1459-1463.

Campbell S (1980). Singular Systems of Differential Equations, London: Pitman.

Cheng P, Deng F, Dai X (2010). Razumikhin-type theorems for asymptotic stability of impulsive stochastic functional differential systems. J. Sys. Sci. Sys. Eng., 19(1): 72-84.

Cobb D (1984). Controllability, observability, and duality in singular systems. IEEE Trans. Autom. Control, 29(12): 1076-1082.

$3350 \quad$ Sci. Res. Essays

Guan ZH, Chan CW, Leung AYT, Chen G (2001). Robust stabilization of singular-impulsive-delayed systems with nonlinear perturbations. IEEE Trans. Circuits Syst., 48(8): 1011-1019.

Guan ZH., Liu YQ, Wen XC (1995). Decentralized stabilization of singular and time-delay large scale control systems with impulsive solutions. IEEE Trans. Autom. Control., 40(8): 1437-1441.

Guan ZH., Yao J, Hill DJ (2005). Robust $H_{\infty}$ control of singular impulsive systems with uncertain perturbations. IEEE Trans. Circuits Syst.II 52(6): 293-298.

Ishihara JY, Terra MH (2002). On the Lyapunov theorem for singular systems. IEEE Trans. Autom. Control, 47(11): 1926-1930.

La Salle JP, Lefschetz S (1961). Stability by Lyapunov's Direct Method with Applications. New York: Academic Press.

Liu L, and Sun J (2008). Finite-time stabilization of linear systems via impulsive control. Int. J. Control 81: 905-909.

Pachpatte BG (1998). Inequalities for Differential and Integral Equations. New York: Academic Press.

Wang CJ, Lia HE (2001). Impulse observability and impulse controllability of linear time-varying singular systems. Automatica, 37(11): 1867-1872.
Wang Q, Liu X (2007). Exponential stability of impulsive cellular neural networks with time delay via Lyapunov functionals. Appl. Math. Comput.,194(1): 186-198.

Weiss L, Infante EF (1967). Finite time stability under perturbing forces and on product spaces. IEEE Trans. Autom. Control., 12(11): 54-59.

Yang T (2001). Impulsive Control Theory. Berlin, Germany: SpingerVerlag.

Yao J, Guan ZH, Chen GR, Ho DWC (2006). Stability, robust stabilization and $H_{\infty}$ control of singular-impulsive systems via switching control. Syst. Control Lett., 55(11): 879-886.

Zhang Y, Sun J (2005). Stability of impulsive neural networks with time delays. Phys. Lett., A 348(1-2): 44-50.

Zhao S, Sun J, Liu L (2008). Finite-time stability of linear time-varying singular systems with impulsive effects. Int. J. Control 81: 1824-1829. 